\title{
Transient Stability Assessment of Power Systems in the Presence of Shunt Compensators Using Trajectory Sensitivity Analysis
}

\author{
Amin Nasri, Student Member, IEEE, Mehrdad Ghandhari, Member, IEEE, Robert Eriksson, Member, IEEE,
}

\begin{abstract}
Trajectory sensitivity analysis (TSA) is used as analysis tool for suitable placement of shunt compensators in the power system. The goal is to maximize the benefit of these devices in order to enhance the transient stability of the system. For this purpose, the trajectory sensitivities of the rotor angles of generators with respect to the reactive power injected into different nodes of the system are calculated in the presence of most probable severe faults. Based on the obtained trajectory sensitivities, a method is proposed to determine how effective the shunt compensation in each node is for improving the transient stability. This method is applied to the IEEE 3-machine 9-bus to find the priorities of system's nodes for installation of shunt compensators. Simulation with industrial software shows the validity and efficiency of the proposed method.
\end{abstract}

Index Terms-Trajectory Sensitivity Analysis (TSA), Static Synchronous Compensator (STATCOM), Transient Stability, Critical Clearing Time (CCT).

\section{INTRODUCTION}

$\mathbf{T}$ RANSIENT stability is the ability of power system to keep its synchronism when a large disturbance, like three phase short circuit, occurs in the system. Nowadays, deregulation in electricity markets, increasing electricity demands and high penetration of renewable energy sources in one hand and economic and environmental constraints on installing new transmission lines and building new power plants on the other hand, have pushed the existing transmission systems to be operated close to their critical conditions. So, there is a higher risk of transient instability in today's heavy-loaded and interconnected power systems.

Flexible AC Transmission System (FACTS) devices can have a significant impact on operational flexibility and controllability of the power system. Using these devices, the power flow through the system can be controlled dynamically in a way to increase the transient stability margins and make the system more secure. Since the impact of these devices on the system's stability is strongly dependent on their locations, there is a great need for developing an analytical tool to provide useful information to the system planners regarding the best possible installation locations of them. In this paper, an effective tool is developed to identify the most suitable places to install shunt compensators to improve transient stability of power system. Assessment of rotor angle stability is essential to study the dynamic behaviour of the power system. Time domain simulation is the traditional way for transient stability assessment which has two main disadvantageous, namely time-consuming computation requirement and incapability to provide any information regarding the stability margin [1]. The other method

A. Nasri, M. Ghandhari and R. Eriksson are with the Department of Electric Power Systems, School of Electrical Engineering at KTH Royal Institute of Technology, Sweden, e-mail: (see http://www.kth.se/en/ees/omskolan/organisation/avdelningar/eps/abouteps). which has been widely used for this purpose is transient energy function (TEF) method [2]-[4]. The significant advantage of this method is its capability to provide a stability index [1]. Several methodologies have been proposed based on the sensitivity of TEF to determine the effectiveness of FACTS devices to improve system's transient stability [5], [6]. Despite all the advantages of the TEF based methods, the main shortcoming of them is their high complexity in the following situations: (i) considering differential-algebraic equation (DAE) models of power systems, (ii) dealing with the detailed models of the system's components, (iii) when a number of system's parameters have to be taken into account for the sensitivity analysis [7]-[11].

Applications of trajectory sensitivity analysis (TSA) have been introduced as an alternative to overcome the mentioned shortcoming of the TEF based method [7]. Ref. [8] has used TSA to calculate the critical values of some power system's parameters such as fault clearing time, mechanical input power of generators, etc. The proposed method in [8] is based on the computation of the norm of trajectory sensitivities of rotor angles and speeds of generators with respect to the parameters of interest. Ref. [9], [10] has discussed the application of TSA to power systems containing series and shunt compensators. A transient stability index has been introduced based on the numerical formulation of TSA and has been calculated for the power system with different locations of series and shunt compensators. Using numerical formulation of TSA, considering the compensators' models in the study and simulating the power system for all the possible locations of compensators has caused high computational burden for the proposed method in [9], [10]. Ref. [11] has developed a multi-parameter trajectory sensitivity approach to find the best locations of series compensators in order to improve the transient stability. An index of proximity to instability has been determined based on the norm of the trajectory sensitivities of the rotor angles and the speeds of generators with respect to the transmission line susceptances. Using the analytical formulation of TSA in [11] and also in this paper, the cumbersome computational process becomes much simpler compared to the numerical method in [8], [9]. The latter will be explained later.

In this paper, a novel method is proposed based on the analytical formulation of TSA to determine the most appropriate places of shunt compensations to amend the transient stability of power system. Trajectory sensitivities of the rotor angles of generators are computed directly with respect to the reactive power injected into different nodes of the system. Based on the obtained trajectory sensitivities, the most effective locations to install shunt compensators are found. The major contribution of this paper is to use the analytical formulation of TSA 
for the suitable placement of shunt compensators in order to enhance the transient stability which is computationally more efficient than the method described in $[9,10]$. The system under consideration is IEEE 3-machine 9-bus system in this paper.

\section{Power SYSTEM MOdeling AND TRAJECTORY SENSITIVITY ANALYSIS}

As explained comprehensively in [12], power systems can be modeled by the following differential algebraic equations

$$
\begin{gathered}
\underline{\dot{x}}=\underline{f}(\underline{x}, y) \\
0= \begin{cases}g^{-}(\underline{x}, y) & s(\underline{x}, y)<0 \\
g^{+}(\underline{x}, y) & s(\underline{x}, y)>0\end{cases} \\
\underline{x}=\left[\begin{array}{l}
x \\
\lambda
\end{array}\right] \quad \underline{f}=\left[\begin{array}{l}
f \\
0
\end{array}\right] \\
\underline{x}\left(t_{0}\right)=\underline{x}_{0}, y\left(t_{0}\right)=y_{0}
\end{gathered}
$$

where $\underline{x}$ is a vector containing the dynamic states $x$ and the system parameters $\lambda$, and $y$ is a vector of algebraic states. Rotor angles of the generators, impedances of the transmission lines and magnitude and angle of bus voltages are the examples of the dynamic states, power system parameters and algebraic states, respectively. $\underline{x}_{0}$ and $y_{0}$ are the initial conditions of the mentioned vectors. Function $f$ is the set of differential equations which model the dynamics of equipments like generators. Events like a three phase short circuit fault occurs when $s(\underline{x}, y)=0$. The algebraic equations $g$ consist of the network equations based on Kirchhoff's current law, i.e. the sum of all current (or powers) flowing into each bus must be equal to zero, $g^{-}$and $g^{+}$show the algebraic equations before and after occurrence of events.

To calculate the trajectory sensitivities analytically, the derivatives of (1), (2) are calculated with respect to $\underline{x}_{0}$ which contains both the dynamic state's initial conditions and also systems' parameters

$$
\begin{gathered}
\underline{\dot{x}}_{\underline{x}_{0}}=\underline{f}_{\underline{x}}(t) \underline{x}_{\underline{x}_{0}}+\underline{f}_{y}(t) y_{\underline{x}_{0}} \\
0=g_{\underline{x}}(t) \underline{x}_{\underline{x}_{0}}+g_{y}(t) \underline{y}_{\underline{x}_{0}}
\end{gathered}
$$

The system is assumed to be away from the events. The initial conditions for $\underline{x}_{x_{0}}$ and $y_{\underline{x}_{0}}$ are obtained by differentiating (3) with respect to $\underline{x}_{0}$. It is clear that the initial value for the trajectory sensitivities of dynamic states is an identity matrix. Using this identity matrix, the initial values for the trajectory sensitivities of algebraic states can be also computed from (8).

$$
\underline{x}_{\underline{x}_{0}}\left(t_{0}\right)=I, \quad y_{\underline{x}_{0}}\left(t_{0}\right)=-\left(g_{y}\left(t_{0}\right)\right)^{-1} g_{\underline{x}_{0}}\left(t_{0}\right)
$$

$\underline{f}_{x}, \underline{f}_{y}, g_{\underline{x}}$ and $g_{y}$ are time varying functions which are calculated along the system trajectories. When an event occurs in the system, jump condition should be derived for computation of the trajectory sensitivities which is fully described in [12]. To find the trajectory sensitivities, the DAEs (1)-(2) and (5)(6) will be solved simultaneously with the initial conditions described above using trapezoidal integration technique.

To calculate the trajectory sensitivity of state variable $x$ to the parameter $\lambda$ with a numerical formulation of TSA, a small perturbation of $\triangle \lambda$ over the nominal parameter $\lambda_{0}$ should be considered such that $\lambda=\lambda_{0}+\triangle \lambda$. So, the numerical estimation of sensitivity is defined as $x_{\lambda}=\left(x(\lambda)-x\left(\lambda_{0}\right)\right) / \triangle \lambda$.

\section{TRANSIENT STABILITY ASSESSMENT USING TRAJECTORY SENSITIVITY ANALYSIS}

Power systems may become transiently unstable after being subjected to large disturbances. The result of transient instability appears in the form of increasing rotor angles of some generators which leads to their loss of synchronism with other generators. So, monitoring angular swings of generators (or the equivalent angle of all the generators) could be one way to check the transient stability of power system. To improve the transient stability, power system parameters can be controlled (if applicable) in a way to have positive effects on the variation of rotor angles of generators when the system is subjected to a fault and prevent power system from being unstable.

Shunt FACTS devices like Static Synchronous Compensator (STATCOM) are capable of injecting (absorbing) reactive power to (from) the power system's nodes. They can control dynamically the amount of this reactive power injection (absorbtion) so that to improve the rotor angle stability of power system. Due to the heavy costs of these devices, it is necessary to find the appropriate locations to install minimum numbers of them needed for the stability enhancement. In this paper, trajectory sensitivities of rotor angles with respect to the reactive power injected into the different nodes of the system are used for suitable placement of shunt compensators.

So, the matrices of system parameters and trajectory sensitivities of dynamical states to these parameters are as follows

$$
\begin{gathered}
\lambda=\left[\begin{array}{llll}
Q_{i n j_{1}} & Q_{i n j_{i}} & \ldots & Q_{i n j_{N}}
\end{array}\right] \\
\frac{\partial \delta}{\partial \lambda}=\left[\begin{array}{cccc}
\frac{\partial \delta_{1}}{\partial Q_{i n j_{1}}} & \frac{\partial \delta_{1}}{\partial Q_{i n j_{i}}} & \ldots & \frac{\partial \delta_{1}}{\partial Q_{i n j_{N}}} \\
\frac{\partial \delta_{2}}{\partial Q_{i n j_{1}}} & \frac{\partial \delta_{2}}{\partial Q_{i n j_{i}}} & \cdots & \frac{\partial \delta_{2}}{\partial Q_{i n j_{N}}} \\
\cdot & \cdot & \cdot & \cdot \\
\cdot & \cdot & \cdot & \cdot \\
\cdot & \cdot & \cdot & \cdot \\
\frac{\partial \delta_{n}}{\partial Q_{i n j_{1}}} & \frac{\partial \dot{\delta}_{n}}{\partial Q_{i n j_{i}}} & \ldots & \frac{\partial \delta_{n}}{\partial Q_{i n j_{N}}}
\end{array}\right]
\end{gathered}
$$

where $Q_{i n j_{i}}$ is the reactive power injected to the $i^{\text {th }}$ node, $N$ is the number of nodes, $\delta_{j}$ is the rotor angle of the $j^{t h}$ generator, and $n$ is the number of generators. The matrix (9) is a part of solution to the (5) described in Section II.

\section{APPROPRIATE PLACEMENT OF SERIES COMPENSATORS TO IMPROVE TRANSIENT STABILITY}

\section{A. Definition of equivalent angle $\delta_{e q_{t}}$}

In this part, an equivalent angle is defined and the trajectory sensitivity of this angle to the the reactive power injected into the different nodes of the system is introduced for appropriate placement of shunt compensators. To define this angle, machines are separated to two groups named $A$ and $B$ depending if their rotor angles $(\delta)$ after fault occurrence, in center of inertia (COI) reference, are accelerating or decelerating, respectively. Then, these two groups are replaced by a single machine equivalent system. The equivalent angle $\delta_{e q_{t}}$ includes all the rotor angles and is defined as follows [13]

$$
M_{A}=\sum_{i \in A} M_{i}, \quad M_{B}=\sum_{j \in B} M_{j}
$$




$$
\begin{gathered}
\delta_{A}=M_{A}^{-1} \sum_{i \in A} M_{i} \delta_{i}, \quad \delta_{B}=M_{B}^{-1} \sum_{j \in B} M_{j} \delta_{j} \\
\delta_{e q_{t}}=\delta_{A}-\delta_{B}
\end{gathered}
$$

where $M_{i}$ and $\delta_{i}$ are the inertia and rotor angle of the $i^{\text {th }}$ generator, respectively. According to this definition, the trajectory sensitivity of the equivalent angle to the reactive power injected to different nodes of the system is calculated as follows

$$
\begin{aligned}
\frac{\partial \delta_{A}}{\partial Q_{i n j_{i}}} & =M_{A}^{-1} \sum_{i \in A} M_{i} \frac{\partial \delta_{i}}{\partial Q_{i n j_{i}}} \\
\frac{\partial \delta_{B}}{\partial Q_{i n j_{i}}} & =M_{B}^{-1} \sum_{j \in B} M_{j} \frac{\partial \delta_{j}}{\partial Q_{i n j_{i}}} \\
\frac{\partial \delta_{e q_{t}}}{\partial Q_{i n j_{i}}} & =\frac{\partial \delta_{A}}{\partial Q_{i n j_{i}}}-\frac{\partial \delta_{B}}{\partial Q_{i n j_{i}}}
\end{aligned}
$$

B. The proposed algorithm for appropriate placement of shunt compensators to improve transient stability

- Most severe faults with the following clearing times are selected.

$$
t_{c l_{i}}=t_{c c_{i}}-\varepsilon
$$

where $t_{c l_{i}}$ and $t_{c c_{i}}$ are the clearing time and critical clearing time (CCT) of the $i^{\text {th }}$ fault respectively, and $\varepsilon$ is a positive small number ( $1 \mathrm{~ms}$ in this paper).

- For each fault:

1) (1)-(2) and (5)-(6) are solved simultaneously using the mentioned $\lambda$ vector and all the dynamic and algebraic variables and their trajectory sensitivities with respect to the parameter vector $\lambda$ are calculated. Simulation time does not need to be so long (first swing only).

2) Based on the obtained data for the rotor angles $(\delta)$ of the generators, generators are divided to $A$ and $B$ groups which oscillate against each other.

3) The matrix of trajectory sensitivities of dynamical states (9) is obtained and based on the definition of $\delta_{e q_{t}}$, trajectory sensitivities of $\delta_{e q_{t}}$ with respect to the reactive power injected into different nodes of the system are computed.

4) The maximum peak to bottom value of trajectory sensitivity of $\delta_{e q_{t}}$ to each injected reactive power into nodes (except the faulty node) after fault occurrence are determined.

5) The values obtained in 4) are normalized to their maximum value and are divided to the clearing time of the fault.

- Finally

1) for each node, sum of normalized trajectory sensitivities of $\delta_{e q_{t}}$ to the injected reactive power into that node for different faults is calculated (data from second step). These results show how effective each node is for improvement of transient stability.

2) The nodes which have the most positive effects are chosen for installation of shunt compensators.

\section{Simulation AND RESUlts}

The IEEE 3-machine 9-bus test system is used to evaluate the proposed algorithms. The system is shown in Fig. 1. The system data is taken from [14]. The reactances of transformers are included in transient reactances of generators. Classical model is used for the generators. Table I shows list of the selected faults which are applied to this test system. When a fault occurs in the system, generators are divided to two transient groups as mentioned in section IV. A. Fig. 2 shows the rotor angles of generators in center of inertia reference for fault 6 . It is clear that generators 2 and 3 are accelerating and generator 1 is decelerating. For this fault, generator 1 is selected as the $B$ group and generators 2 and 3 as the $A$ group, and equivalent rotor angle is calculated based on this classification and is also depicted in Fig. 2. The trajectory sensitivities of $\delta_{e q_{t}}$ with respect to the the reactive power injected into different nodes of the system are calculated and shown in Fig. 3. This figure shows that the signs of trajectory sensitivities of $\delta_{e q_{t}}$, after fault occurrence, with respect to the reactive power injected into different nodes of the system are positive. Nodes 3, 5, 4, 2 and 1 have the largest absolute peak to bottom value of trajectory sensitivities, respectively.

It is so important to understand the meaning of these absolute peak to bottom values and their signs after occurring faults. It can be written based on the trajectory sensitivities

$$
\triangle \delta_{e q_{t}} \approx\left(\frac{\partial \delta_{e q_{t}}}{\partial Q_{i n j_{i}}}\right) \triangle Q_{i n j_{i}}
$$

The sign of trajectory sensitivities determines if the reactive power should be injected to or absorbed from different nodes

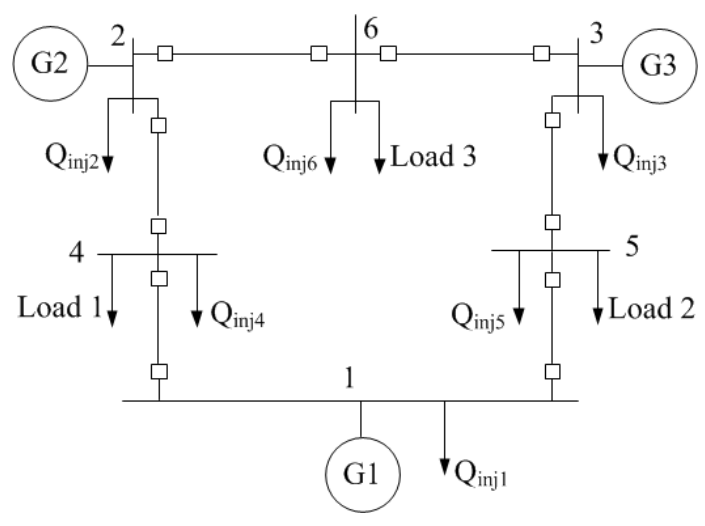

Figure 1. Modified IEEE 9-bus 3-machine test system.

Table I

List of selected faults - IEEE 9-bus 3-machine test system

\begin{tabular}{|c|c|c|}
\hline Name & Fault Location & Clearing Time \\
\hline Fault 1 & Line 1-4 very close to bus 1 & $143 \mathrm{~ms}$ \\
\hline Fault 2 & Line 2-6 very close to bus 2 & $142 \mathrm{~ms}$ \\
\hline Fault 3 & Line 3-5 very close to bus 3 & $129 \mathrm{~ms}$ \\
\hline Fault 4 & Line 4-2 very close to bus 4 & $202 \mathrm{~ms}$ \\
\hline Fault 5 & Line 1-5 very close to bus 5 & $195 \mathrm{~ms}$ \\
\hline Fault 6 & Line 6-3 very close to bus 6 & $191 \mathrm{~ms}$ \\
\hline
\end{tabular}




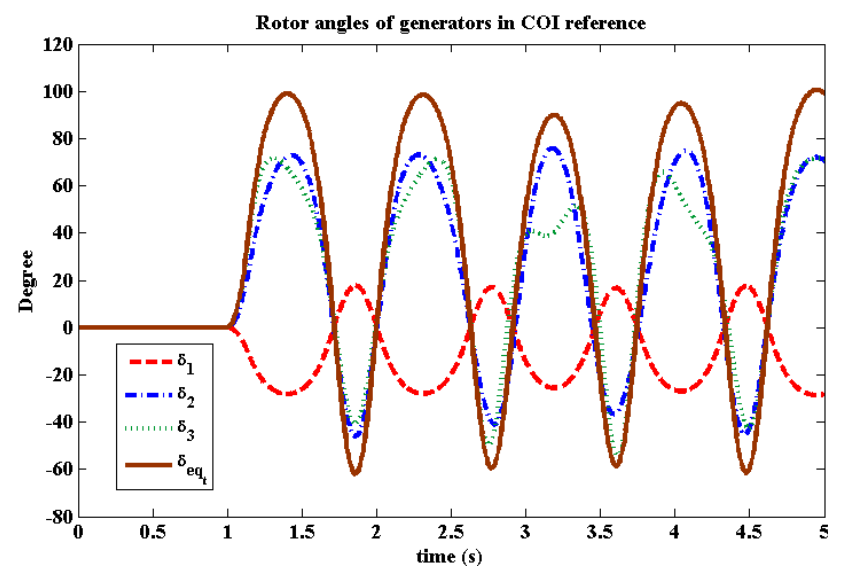

Figure 2. Rotor angles of the generators and the equivalent angle $\left(\delta_{e q_{t}}\right)$.

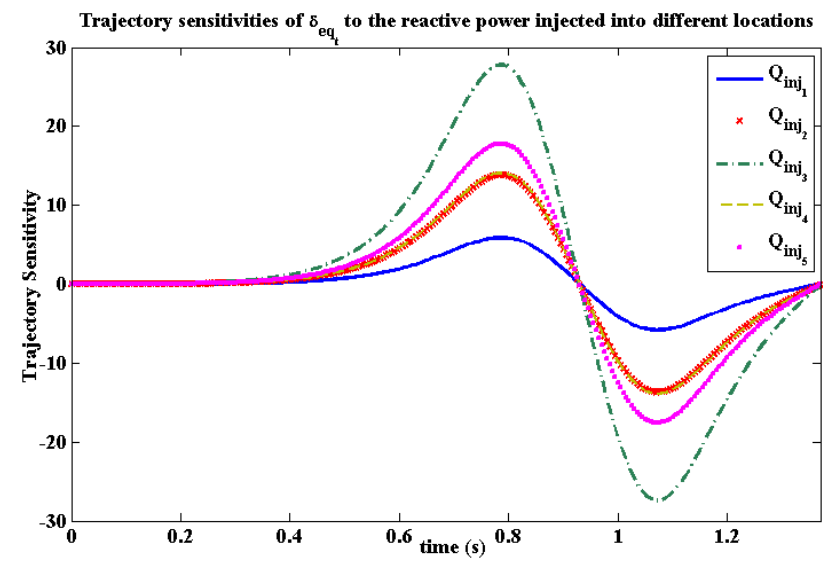

Figure 3. Trajectory sensitivities of $\delta_{e q_{t}}$ to the reactive power injected into the different nodes of the system for Fault 6

of system for the stability enahncement. Since STATCOM is going to be installed in the system, $\triangle Q_{i n j_{i}}$ could be both positive and negative. Due to the sharp increase of $\delta_{e q_{t}}$ after fault occurrence which is visible from Fig. $2, \triangle \delta_{e q t}$ should be negative to decrease $\delta_{e q_{t}}$, and improve the transient stability. For this fault, it can be seen form Fig. 3 that the values of trajectory sensitivities of $\delta_{e q_{t}}$ with respect to the reactive power injected into all the nodes $\left(\partial \delta_{e q_{t}} / \partial Q_{i n j_{i}}\right)$ are positive. So, $\triangle Q_{i n j_{i}}$ should be negative to make $\triangle \delta_{e q_{t}}$ also negative which means that the reactive power should be injected to the nodes after fault occurrence (considering the direction of $Q_{i n j_{i}}$ shown in Fig. 1).

The absolute peak to bottom value of trajectory sensitivities also shows how sensitive $\delta_{e q_{t}}$ is to the shunt compensation in different nodes. For this fault, it means that for the same amount of $\triangle Q_{i n j_{i}}$, there will be larger value of $\triangle \delta_{e q_{t}}$ when STATCOM is placed at Nodes 3, 5, 4, 2 and 1, respectively. So, the most appropriate place to install the STATCOM to improve the transient stability of this test system corresponding to this fault is node 3 . The same calculations are done for the other faults and results are given in Table II. For each fault, computed peak to bottom values of trajectory sensitivities are normalized to their maximum value. Each row corresponding to each fault shows these normalized trajectory sensitivities of $\delta_{e q t}$. It can be seen that one of the trajectory sensitivities is equal to 1 for each row which shows the most effective place to install shunt compensator for improving the transient stability corresponding to that fault. The next step is to consider clearing time of each fault. For this purpose, all the digits of each row are divided to the clearing time of its corresponding fault. The final step is to calculate the total effect of injecting reactive power into each node on the transient stability for all the selected faults and decide about the best location for installing shunt compensators. According to the obtained results shown in Table III, Node 6 is the best location for installing shunt compensator considering all the selected faults.

Table II

Normalized trajectory sensitivities of $\delta_{e q_{t}}$ for different fault scenarios

\begin{tabular}{|l|c|c|c|c|c|c|}
\hline & Node 1 & Node 2 & Node 3 & Node 4 & Node 5 & Node 6 \\
\hline Fault 1 & - & 0,5493 & 0,7475 & 0,9280 & $\mathbf{1 , 0 0 0 0}$ & 0,7974 \\
\hline Fault 2 & 0,2870 & - & 0,9107 & 0,6517 & 0,7816 & $\mathbf{1 , 0 0 0 0}$ \\
\hline Fault 3 & 0,2520 & 0,6486 & - & 0,5967 & 0,6682 & $\mathbf{1 , 0 0 0 0}$ \\
\hline Fault 4 & 0,3084 & 0,6820 & 0,9693 & - & 0,9324 & $\mathbf{1 , 0 0 0 0}$ \\
\hline Fault 5 & 0,2853 & 0,6804 & 0,9938 & 0.7448 & - & $\mathbf{1 , 0 0 0 0}$ \\
\hline Fault 6 & 0,2412 & 0,5054 & $\mathbf{1 , 0 0 0 0}$ & 0,5794 & 0,7016 & - \\
\hline
\end{tabular}

Table III

Total effects of reactive power injection into each node on the transient stability

\begin{tabular}{|l|c|c|c|c|c|c|}
\hline & Node 1 & Node 2 & Node 3 & Node 4 & Node 5 & Node 6 \\
\hline Sum & 8,2339 & 18,4217 & 26,8355 & 22,9648 & 26,0348 & $\mathbf{3 0 , 4 8 8 4}$ \\
\hline
\end{tabular}

To calculate these sensitivities based on the numerical formulation of TSA described in section II, $(n+1) \times n_{f}$ time domain simulations should be run to to find the nominal and perturbed trajectories for a power system with $n$ nodes (possible places to install shunt compensator) and $n_{f}$ fault scenarios. For this test system which has 6 nodes and 6 fault scenarios, the required number of time domain simulations has been reduced from 42 $\left((n+1) \times n_{f}\right)$ to $6\left(n_{f}\right)$ because of the analytical formulation of TSA. For the large-scale power systems, the proposed method simplifies significantly the cumbersome computational process of placement procedure.

\section{Simulation With INDUSTRIAL SOFTWARE}

To verify the results, industrial software SIMPOW ${ }^{\circledR} 11$ is used to simulate the test system in the presence of STATCOM. An injection model of STATCOM has been implemented and placed in different locations and the new CCTs of the selected faults have been calculated for each location using time domain simulations. For a better comparison, the STATCOM controller injects dynamically the same amount of reactive power for different placement scenarios. Fig. 4 shows the variation of equivalent rotor angle after fault occurrence at node 6 for different locations of STATCOM. It is clear that the non-compensated system is transiently unstable. As it was expected from Table II, shunt compensation of Nodes 3, 5, 4, 


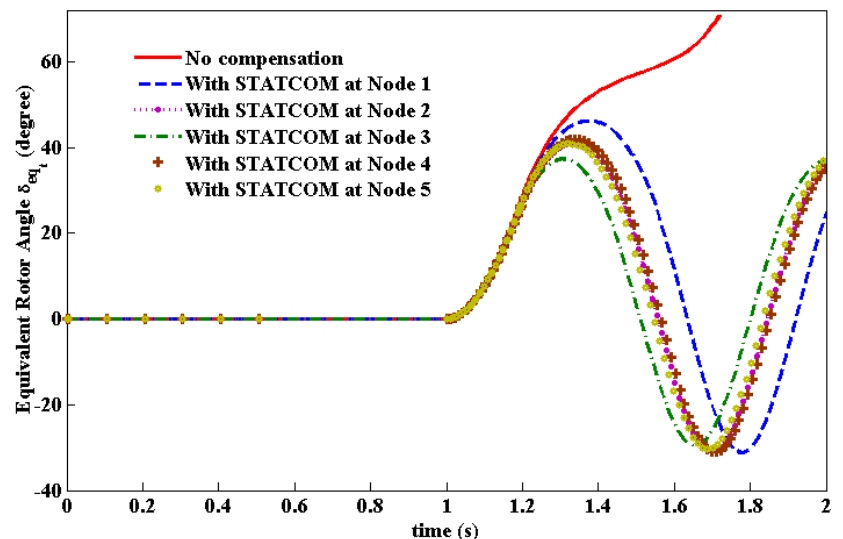

Figure 4. Fault at bus 6 with a clearing time $=193 \mathrm{~ms}-$ Equivalent rotor angle, $\delta_{e q_{t}}$, for different locations of STATCOM

Table IV

Difference between new and old CCTs (ms) of faults after installing STATCOM in different nodes

\begin{tabular}{|l|c|c|c|c|c|c|}
\hline & Node 1 & Node 2 & Node 3 & Node 4 & Node 5 & Node 6 \\
\hline Fault 1 & - & 15 & 20 & 22 & 22 & 20 \\
\hline Fault 2 & 6 & - & 18 & 10 & 12 & 19 \\
\hline Fault 3 & 4 & 8 & - & 7 & 8 & 13 \\
\hline Fault 4 & 11 & 23 & 38 & - & 35 & 38 \\
\hline Fault 5 & 12 & 30 & 42 & 33 & - & 45 \\
\hline Fault 6 & 10 & 23 & 47 & 23 & 29 & - \\
\hline
\end{tabular}

2 and 1 have the most positive effects on the transient stability, respectively. Table IV shows the difference between new and old CCTs of all the selected faults corresponding to different location of STATCOM. It can be seen that the results are consistent with the normalized trajectory sensitivities shown in Table II. The results confirms that installing STATCOM at Node 6 has the best total impact on the transient stability.

\section{CONCLUSION}

A novel method has been proposed based on the trajectory sensitivity analysis (TSA) to find the suitable locations of shunt compensators in order to improve the transient stability of power system. In this method, trajectory sensitivities of the rotor angles of the generators with respect to the reactive power injected into the different nodes of the system have been computed for different fault scenarios. Using the analytical formulation of TSA and selecting appropriate system's parameters, the number of required time domain simulations have been reduced significantly. Moreover, the proposed method does not need to consider the detailed model of STATCOM for its simulations. The numerical result on the IEEE 3-machine 9-bus system have shown validity, accuracy and efficiency of the proposed approach. The proposed method can also be easily applied to a very large power system assuming the most critical faults are already known. Future research could be the extension of this technique for the placement of multiple FACTS devices with different types to enhance the rotor angle stability and also it's application to a large-scale power system.

\section{ACKNOWLEDGMENT}

Amin Nasri has been awarded an Erasmus Mundus PhD Fellowship. The authors would like to express their gratitude towards all partner institutions within the programme as well as the European Commission for their support.

\section{REFERENCES}

[1] G. A. Maria, C. Tang, J. Kim, "Hybrid transient stability analysis," IEEE Trans. on power systems, vol. 5, pp. 384-393, May 1990.

[2] A. A. Fouad, S. E. Stanton, "Transient Stability of a Multi-Machine Power System. Part II. Critical Transient Energy ," Power Engineering Review, vol. PER-1, pp. 3417-3424, July 1981.

[3] T. Athay, P.Podmore, S.Virmani, "A Practical Method for the Direct Analysis of Transient Stability," IEEE Trans. on power appratus and systems, vol. PAS-98, No. 2, pp. 573-584, March/April 1979.

[4] A. A. Fouad, V.Vittal, T. K. Oh, "Critical Energy for Direct Transeint Stability Assessment of a Multimachine Power System," IEEE Trans. on power appratus and systems, vol. 103, pp. 2199-2206, August 1984.

[5] A. Ishigame, S. Kawamoto, T. Taniguchi, "Structural control of electric power networks for transient stability," IEEE Trans. on power systems, vol. 9, pp. 1575-1581, August 1994.

[6] K. N. Shubhanga, A. M. Kulkarni, "Application of structure preserving energy margin sensitivity to determine the effectiveness of shunt and series FACTS devices," IEEE Trans. on power systems, vol. 17, pp. 730-738, August 2002.

[7] M. J. Laufenberg, M. A. Pai, "A new approach to dynamic security assessment using trajectory sensitivities," IEEE Trans. on power systems, vol. 13, pp. 953-958, August 1998

[8] T. B. Nguyen, M. A. Pai, "Sensitivity approaches for direct computation of critical parameters in a power system," International Journal of Electrical Power \& Energy Systems, vol. 24, pp. 337-343, June 2002.

[9] D. Chatterjee, A. Ghosh, "Transient Stability Assessment of Power Systems Containing Series and Shunt Compensators," IEEE Trans. on power systems, vol. 22, pp. 1210-1220, August 2007.

[10] D. Chatterjee, A. Ghosh, "Improvement of transient stability of power system with STATCOM-controller using trajectory sensitivity," Electrical Power and Energy Systems, vol. 33, pp. 531-539, January 2011.

[11] A. Zamora-Cárdenas, C. R. Fuerte-Esquivel, "Multi-parametertrajectory sensitivity approach for location of series-connected controllers to enhance power system transient stability," Electric Power Systems Research, vol. 80, pp. 1096-1103, March 2010.

[12] I. A. Hiskens, M. A. Pai, "Trajectory sensitivity analysis of hybrid systems," IEEE Trans. circuits and systems, vol. 47, February 2000.

[13] M. Pavella, D. Ernst, d. Ruiz-Vega, "Power System System Transient Stability Analysis and Control," Kluwer Academic Publishers, 2000.

[14] M. A. Pai, "Energy Function Analysis for Power System Stability," Appendix A, 2007.

Amin Nasri received his M.Sc. degree in Electrical Engineering from the Sharif University of Technology, Tehran, Iran, in 2008. He is pursuing the Erasmus Mundus Joint Doctorate in Sustainable Energy Technologies and Strategies (SETS) hosted by Comillas Pontifical University, Spain; Royal Institute of Technology, Sweden; and Delft University of Technology, Netherlands. $\mathrm{He}$ is currently a Ph.D. Student in the Division of Electric Power Systems, School of Electrical Engineering at KTH Royal Institute of Technology.

Mehrdad Ghandhari received the M.Sc., Tech. Lic. and Ph.D. degrees in Electrical Engineering from Royal Institute of Technology, Stockholm, Sweden, in 1995, 1997, and 2000, respectively. He is currently Associate Professor at KTH Royal Institute of Technology.

Robert Eriksson received his M.Sc., Tech. Lic. and Ph.D. degrees in Electrical Engineering from KTH Royal Institute of Technology, Stockholm, Sweden, in 2005, 2008 and 2011 respectively. He is currently a Postdoctoral researcher in the Division of Electric Power Systems, School of Electrical Engineering at KTH Royal Institute of Technology. 